\title{
EL TRANSMEDIA AUDIOVISUAL EN EL AULA UNIVERSITARIA.
}

\author{
Audiovisual Transmedia at University.
}

\author{
Autores: ANDREU-SÁNCHEZ, Celia y MARTÍN-PASCUAL, Miguel Ángel \\ Doctora - Universitat Autònoma de Barcelona-España-celia.andreu@uab.cat \\ DEA-Universitat Autònoma de Barcelona-España-miguelangel.martin@uab.cat
}

\section{Resumen}

Asistimos a una nueva manera de distribuir los contenidos a través de múltiples plataformas. En el contexto actual, la narración ha dejado de producirse en exclusiva para un medio audiovisual concreto, para empezar a hacerlo a través de varios y complementarios medios o plataformas. Por ello, el diseño de este tipo de narrativas debe ser incluido en los proyectos docentes universitarios que trabajen con estudiantes del área de la Comunicación Audiovisual, la Publicidad y el Periodismo. Presentamos un caso de estudio real en el que los alumnos de una asignatura de cuarto curso del grado de Comunicación Audiovisual en la Universitat Autònoma de Barcelona, diseñan y producen una narrativa transmedia de ficción con distribución de contenidos a través de web, red social, televisión y aplicación móvil propia. El resultado de este proyecto es la distribución profesional de diez narrativas audiovisuales transmedia, realizadas por estudiantes universitarios y dirigidas a prosumidores reales actuales.

\section{Palabras clave}

Transmedia, narrativas multiplataforma, aplicaciones móviles, iOS, prosumidor.

\begin{abstract}
A new way to distribute the contents across multiple platforms is taking place in audiovisual industry. In the current context, the story has stopped of being produced exclusively to a particular audiovisual platform, to start doing through various and complementary media or platforms. Therefore, the design of such narratives should be included in university teaching projects that work with students in the area of Audiovisual Communication, Advertising and Journalism. We present a real case study in which students in a fourth-year subject of the degree of Audiovisual Communication at Universitat Autònoma de Barcelona, design and produce a transmedia narrative fiction content with distribution through web, social networking, television and mobile application. The result of this project is the professional distribution of ten transmedia narratives, performed by university students and aimed at actual current prosumers.
\end{abstract}

\section{Key words}

Transmedia, multiplatform narratives, mobile apps, iOS, prosumer. 


\section{Introducción}

Los nuevos modos de narrar ofrecen la posibilidad de ampliar los modelos de distribución audiovisual, pasando del modelo tradicional al fragmentado. Henry Jenkins (2003:1) ofrece un término adaptado para la convergencia de los medios acontecida desde la década de los 90. En su origen, Jenkins critica la falta de compromiso por parte de la industria audiovisual hacia esta nueva manera de distribuir contenidos. Según Jenkins, hace años que la tecnología está preparada para emitir el audiovisual de manera fragmentada a través de diversos medios, sin embargo, los grandes distribuidores de contenidos, la industria del entretenimiento, ha esperado para utilizar esta vía de emisión, prefiriendo seguir con los modelos tradicionales de producción y distribución de contenidos audiovisuales (Jenkins, 2003:1).

Próximo al concepto de transmedia ideado por Jenkins, encontramos otros muchos conceptos y términos que, lejos de facilitar la comunicación, confunden el ámbito teórico y práctico a partes iguales: crossmedia, multiplataforma, intermedia, adaptaciones, entre otros muchos. Ante esta situación, la definición de Carlos Scolari (2013) está cobrando protagonismo en el ámbito científico y aclara bastantes ambigüedades.

Scolari, define la narrativa transmedia "como un tipo de relato donde la historia se despliega a través de múltiples medios y plataformas de comunicación y, donde una parte de los consumidores asume un rol activo en ese proceso de expansión" (Scolari, 2013:46). En esta propuesta encontramos varias ideas de gran relevancia que aplicamos en el proyecto que presentamos: por un lado, la diferenciación entre la distribución de un mismo contenido a través de diferentes plataformas (crossmedia) y la fragmentación del contenido narrativo a través de las mismas (transmedia); por otro lado, se presta atención al concepto de audiencia activa, que deja de comportase como consumidora para formar parte de los prosumidora, productora de los contenidos que ella misma consume, y que participa de la expansión de la narrativa a través de los diferentes medios o plataformas. Según este modelo, corroborado actualmente por la praxis audiovisual profesional, las producciones audiovisuales deben dejar de concebirse para el consumidor de un único medio que sólo escucha. Ahora estas pro- ducciones deben ser ideadas para esos participantes de la narración que interactúan a través de segundas pantallas o redes sociales, principalmente.

En este contexto, es necesario situar el concepto citado de prosumidor que, con procedencia alejada del ámbito audiovisual (Toffler, 1980), nos acerca la idea de que los prosumidores son creadores de bienes, servicios o contenidos con la prioridad del consumo propio frente a la venta (Toffler y Toffler, 2006). Esta idea presenta una enorme evolución y desarrollo en el contexto de la comunicación 2.0, en el que se democratiza la producción y, sobre todo, la distribución de contenidos audiovisuales. Kotler (1986) ya había vinculado el concepto de prosumidor al marketing, y actualmente las nuevas tecnologías facilitan la opción de crear y publicar contenido en Internet para ser compartido en las redes sociales (Giurgiu y Bârsan, 2008). Poco a poco, además, se está comprobando el poder que tienen los consumidores frente a los productores oficiales de contenidos, algo que ya mostró Naomi Klein (1999), cuando evidenció el poder del consumidor frente a la marca.

Las herramientas 2.0 ofrecen una bidireccionalidad capaz de facilitar el "rol activo en el proceso de expansión” del que habla Scolari (2013: 46). Por este motivo, es interesante tener presente que en este contexto de prosumidores, los creadores profesionales de los contenidos audiovisuales transmedia deben diseñar o guiar la interactuación de su audiencia con sus productos.

También, nos encontramos en un contexto donde el espectador deja de situarse en el sofá de su casa de manera exclusiva cuando se enfrenta al consumo de productos audiovisuales. Actualmente, el espectador se encuentra en un entorno de "ubicuidad conectada" (Andreu-Sánchez y Martín-Pascual, 2014a). Esta conexión a la red constante desde en cualquier lugar, permite que se amplíen las audiencias y les facilita el acceso a los contenidos audiovisuales. Esto se produce en paralelo al aumento de penetración de los llamados smartphones o teléfonos inteligentes en nuestro país.

Hoy, España es el país de Europa con mayor tasa de penetración de smartphones (Azevedo y Sánchez, 2013). Datos actualizados en 2013, reflejan que en 
España se descargan 4 millones de aplicaciones para dispositivos móviles al día (The App Date, 2013). Se produce, además, un aumento destacado del consumo de video online desde el teléfono móvil. Azevedo y Sánchez (2013), concluyen que en el año 2012 este aumento de consumo de video fue de un $164 \%$ con respecto al año anterior.

Ante este panorama, es comprensible que el mercado audiovisual deba modernizarse y adaptar su modelo de distribución a una audiencia que es cada vez más activa, prosumidora de productos audiovisuales desde sus dispositivos móviles y con conexión constante a la red.

La formación universitaria y el ámbito educomunicativo no pueden permanecer alejados a esto (Scolari, 2010; Quiroz Velasco, 2010; Kaplún, 2010). En este sentido, son múltiples las experiencias docentes que en los últimos años han planteado el trabajo de las nuevas narrativas audiovisuales en las aulas (Suárez Puerta, 2009; Freitas y Castro, 2010; Martos Núñez y Martos García, 2012; Peñalba García, Pérez Bowie y Noguerol Jiménez, 2013; Hernández, 2014; AndreuSánchez y Martín-Pascual, 2014b; entre otros muchos).

Entre los beneficios más interesantes de estos proyectos de innovación docente, encontramos la incorporación de un marco interdisciplinar que incluye la transmedialidad, la tecnología, la planificación de contenidos, los programas de edición, la convergencia de los medios y su planificación, la interacción del consumidor, la creación de prosumidores y, en algunos niveles de la educación, la alfabetización digital.

\section{Objetivos}

Teniendo en cuenta este panorama, el presente estudio tiene como objetivo principal diseñar un método de trabajo y preparación de una narrativa audiovisual transmedia en el entorno educomunicativo universitario. Concretamente, nos planteamos el objetivo de diseñar, aplicar y analizar una experiencia docente universitaria, de alfabetización transmediática, para que estudiantes de $4^{\circ}$ curso del Grado de Comunica- ción Audiovisual, trabajen para una audiencia prosumidora, que participa de un entorno de ubicuidad conectada.

El proyecto también tiene el objetivo de que los estudiantes creen un proyecto audiovisual transmedia con emisión real, que pueda convertirse en parte de su currículum o "bobina" audiovisual.

\section{Metodología}

Al empezar la asignatura "Géneros interactivos" de $4^{\circ}$ curso del Grado en Comunicación Audiovisual de la Universitat Autònoma de Barcelona, en el curso 2013/2014, se hizo un encargo docente a los estudiantes. El encargo consistía en la producción de un proyecto audiovisual con narración transmedia, entendida según la definición de Scolari (2013). Dicha producción tendría una emisión real y, por tanto, debería ser una producción propia y tener una correcta gestión de los derechos de explotación de las obras audiovisuales de terceros utilizadas. De esta manera, los estudiantes trabajan conmétodo de trabajo profesional para su inserción en el mercado laboral.

La narración debería evolucionar, como mínimo, a través de los siguientes medios o plataformas:

- Una página web, como canal de emisión de base del proyecto.
Una red social, escogida a libre elección de los estudiantes, que se utilizaría para el avance narrativo del proyecto, no como método de promoción. En esta red social, los productores debían prever qué tipo de interactuación haría su público con el proyecto.

La televisión universitaria de la Universitat Autònoma de Barcelona, UAB Campus Media, producida desde la Facultad de Ciencias de la Comunicación. Se trata de una televisión en emisión por IP y con servicio a la carta.

- Una aplicación móvil, que sería diseñada e ideada por los estudiantes, pero programada, en términos técnicos e informáticos, por los docentes. Su publicación se haría en el sistema operativo iOS, propiedad de Apple Inc. 
Además, los estudiantes debían realizar un código QR que colgarían físicamente en un entorno de interés para su público objetivo y que serviría para propiciar el acceso de una línea argumental de su narración a otra, a través de alguna de las plataformas.

Para comprobar la eficacia del proyecto docente, realizamos encuestas cualitativas de calidad de la asignatura a los 19 alumnos que formaron parte de este proyecto y analizamos los resultados obtenidos fruto del trabajo de los estudiantes. La encuesta solicitaba información sobre qué les había gustado del proyecto y qué cambios proponían para el mismo.

La metodología de la experiencia docente diseñada se basa en varias propuestas realizadas en el campo de la pedagogía. Por un lado, se trabajó introduciendo la narración transmediática como un elemento nuevo para transformar la docencia audiovisual existente hasta el momento (Bisquerra,1989). Por otro, se diseñó un marco de investigación-acción (Lewin, 1946) y de aprendizaje basado en problemas o por proyectos (Branda, 2009) en el que los estudiantes tenían que crear una narración audiovisual transmedia. Fruto de este proyecto (o problema) realizarían un aprendizaje colaborativo, interdisciplinar y atendiendo a diversas competencias de manera transversal y activa. El aprendizaje se plantea autodirigido tomando la iniciativa para alcanzar una meta.

\section{Resultados}

Los resultados obtenidos en este estudio de caso se pueden dividir entre los procedentes de los proyectos audiovisuales transmedia que han elaborado los alumnos y los de la experiencia pedagógica propiamente dicha.

\subsection{El proyecto audiovisual transmedia}

El proyecto audiovisual transmedia dio como resultado la creación de diez narraciones transmedia, realizadas por diez grupos de dos estudiantes cada uno, a excepción de uno de los grupos que fue individual, por expreso deseo del estudiante. De esta manera, participaron un total de 19 alumnos. Todos realizaron de manera óptima el proyecto obteniendo resultados académicos notables.

Los estudiantes desarrollaron diez páginas webs distintas para la emisión de cada uno de los proyectos. Las plataformas webs se realizaron haciendo uso de diferentes métodos técnicos y herramientas. Hubo proyectos realizados con Wordpress, Blogger y Wix, principalmente, y un proyecto realizado con un editor de webs profesional.

Las redes sociales utilizadas fueron: Youtube, Google+, Instagram, Twitter y Facebook. Cada grupo trabajó como mínimo con una red en la que sus personajes hacían evolucionar la narración. Cinco de los proyectos hicieron uso de más de una red social.
Los estudiantes encontraron a una audiencia prosumidora que participó de las propuestas activas que su narración planteaba. La participación activa de su audiencia era imprescindible para que el seguimiento del producto audiovisual se consumara.

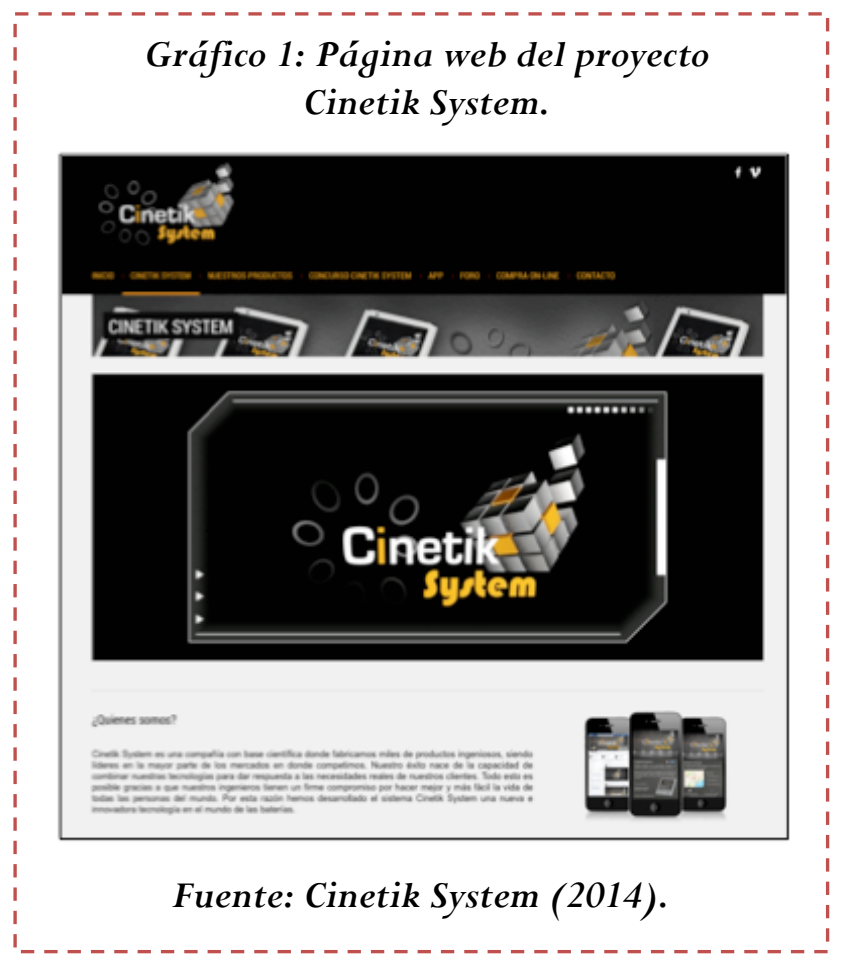

Las narraciones tuvieron distintas emisiones a través de la televisión de la Universitat Autònoma de 
Barcelona, la UAB Campus Media. Para dicha emisión, los alumnos tuvieron que ponerse de acuerdo con los responsables de la televisión y acordar un horario de emisión. Posteriormente, todos los proyectos fueron agrupados en una sección propia, bajo el título de "Projecte Transmèdia", y disponibles para su visionado a la carta. Este espacio sigue disponible (UAB Campus Media, 2014).

En total, los diez grupos han emitido 15 videos en la televisión UAB Campus Media, con emisiones comprendidas desde el 9 de enero al 23 de enero de 2014. Todos los grupos emitieron un video, como se requirió en la asignatura, excepto un proyecto que presentó 6. Este grupo, realizó este trabajo extra por motivación e interés propio, después de que los responsables de la UAB Campus Media les solicitaran más contenidos audiovisuales de su proyecto por el éxito obtenido de audiencia. Esta participación se realizó de manera ajena a la asignatura y las estudiantes de este grupo expresaron su interés y motivación especial por la práctica de esta asignatura que les ofrecía la posibilidad de emitir sus trabajos profesionales por la televisión de la universidad.

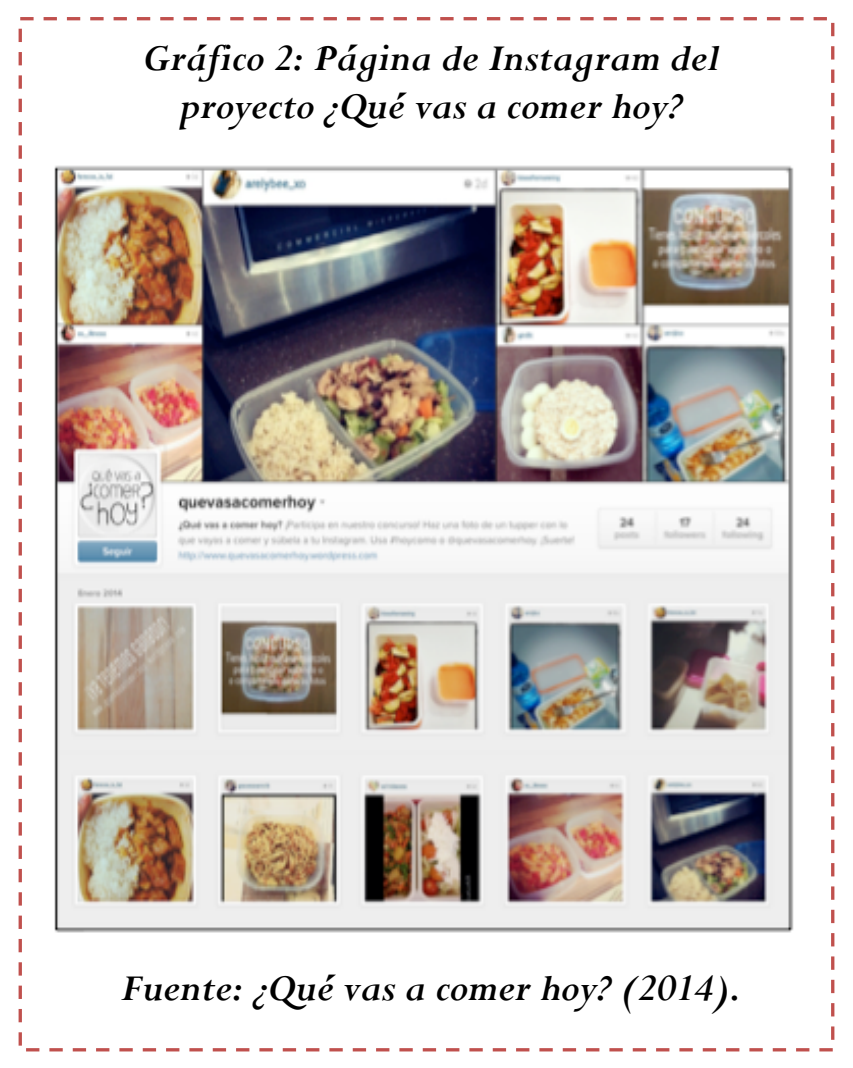

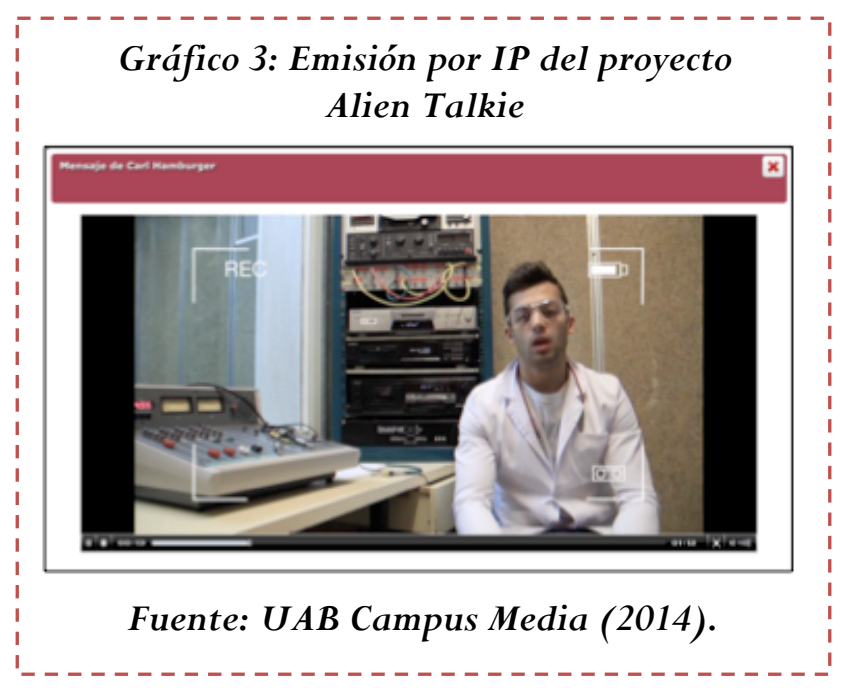

La metodología de trabajo de la aplicación móvil fue la más compleja de todas por existir dependencias alumnos-profesores en la producción de contenidos por parte de los estudiantes, y la producción de la aplicación por parte de los docentes. Realizamos una aplicación, llamada "Transmedia” (iTunes, 2014) para los diez proyectos. En la aplicación el usuario tiene acceso a las diez narrativas de los estudiantes en un momento u otro del desarrollo narrativo. Cada narrativa se encuentra en un punto y tienen un origen y un desenlace narrativo propio. Para facilitar el trabajo de sinergias alumnos-profesores se plantearon cuatro fases de trabajo:

Fase 1. Durante las primeras semanas de trabajo del proyecto, los docentes dan a los estudiantes un espacio en blanco en la interface de la aplicación disponible para que cada grupo diseñe qué desea que aparezca. Cada grupo disponía de dos páginas interactivas.

Fase 2. Una vez los estudiantes han presentado las peticiones de la estructura que desean que tengan sus dos páginas de la aplicación, los docentes les presentamos un mockup o borrador de trabajo de todos los proyectos, siguiendo las líneas formales de trabajo con interfaces de Apple (2014).

Fase 3. En este punto, los alumnos hacen entrega de los materiales audiovisuales ya producidos y los profesores incorporan estos materiales definitivos a la estructura de la aplicación desarrollada en la Fase 2. 
Fase 4. La aplicación ya está completamente creada, pero antes de publicarla, se realiza una fase de test, en la que los estudiantes utilizan la aplicación y detectan posibles errores o presentan peticiones de cambios en la programación.

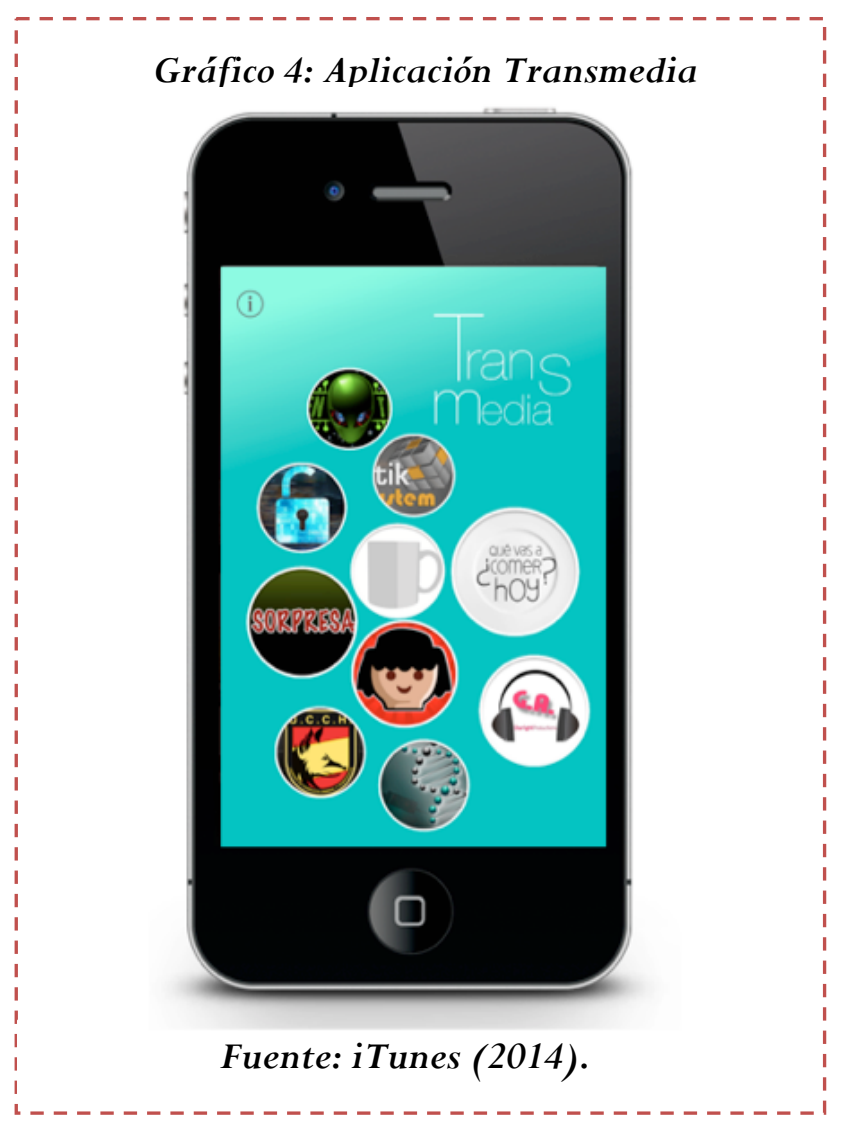

Una vez se han cumplido todas las fases, la aplicación se publica en diciembre de 2013, en la tienda oficial de aplicaciones de Apple, iTunes Store. La aplicación se puede descargar de manera gratuita (iTunes, 2014).

Los resultados del uso del código QR como medio de evolución de su narrativa transmedia fueron dispares. Todos los estudiantes colgaron un póster con un código QR en algún soporte físico (la plaza principal de la universidad, los paneles de información de la facultad, en las calles del barrio de Gracia de Barcelona, en los baños de la universidad, etcétera), sin embargo, varios grupos detectaron que sus audiencias online no tenían acceso físico a los espacios en los que ellos habían publicado el código QR. Para solventar este problema, los estudiantes decidieron incluir los códigos QR en sus narraciones emitidas en web, evitando así perder a una audiencia que no participaría a través del espacio físico del código.

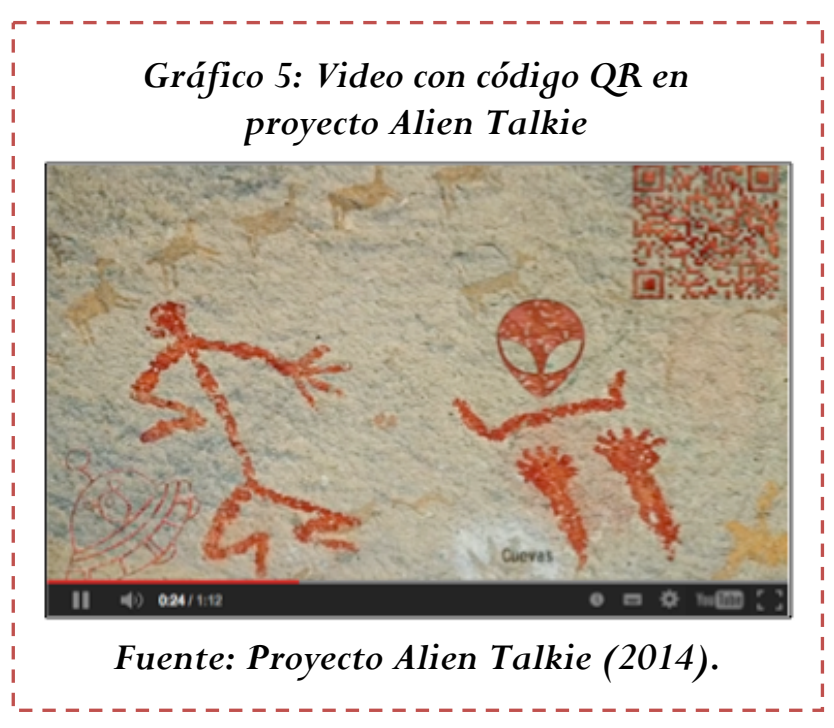

\subsection{El proyecto docente}

Los proyectos transmedia fueron creados de manera exitosa por todos los estudiantes. Trabajaron de manera autónoma durante la mayor parte del semestre. Los docentes les hicieron el encargo en septiembre de 2013 y los alumnos tuvieron que hacer la entrega final del proyecto en enero de 2014. Durante este periodo tuvieron varias tutorías para comprobar la evolución del trabajo, así como varias fechas de entregas para la parte de los proyectos audiovisuales que iban a ser presentadas en la aplicación móvil por motivos evidentes de tiempo ajustado para el desarrollo y la programación de la misma.

En las encuestas realizadas al finalizar la asignatura, los estudiantes valoraron positivamente la libertad temática en la creación de un proyecto de este tipo. Todos los alumnos encuestados coincidieron en que la posibilidad de hacer un proyecto transmedia con emisión y distribución real les permitió explotar su capacidad creativa. La totalidad de menciones positivas en las encuestas implicaban a la metodología del proyecto docente aquí presentado, destacando muy positivamente la emisión real de contenidos audiovisuales realizados con fines didácticos. Las menciones críticas de la asignatura hacían referencia al examen teórico, en ningún caso al proyecto transmedia. 


\section{Conclusiones}

El proyecto aquí presentado obtuvo una realización exitosa y cumplió las metas docentes. Tanto los resultados objetivos de los proyectos audiovisuales transmedia realizados por los estudiantes, como sus opiniones en las encuestas cualitativas realizadas a final de curso, son óptimos. Una de las principales conclusiones que alcanzamos a partir de este trabajo es la necesidad de que los docentes universitarios adecuemos los trabajos audiovisuales requeridos a los alumnos al contexto profesional actual. Este contexto, está protagonizado hoy por el consumo de audiovisuales a través de segundas pantallas y de narraciones multiplataforma.

Cada vez más, las aplicaciones móviles son un modo de comunicación entre los broadcasters y los espectadores. Por ello, entendemos que resulta de gran relevancia diseñar proyectos que no sólo trabajen el audiovisual tradicional, sino que también incluyan la emisión de contenidos (no sólo promoción) a través de la web 2.0 y de plataformas móviles, trabajando desde una perspectiva educomunicativa hacia el prosumidor (Aparici, 2010; Islas, 2010).

Es muy importante tener presente el entorno de ubicuidad conectada en el que se encuentra el consumi- dor. Este acceso a Internet, constante y desde cualquier sitio, provoca que el diseño del los proyectos audiovisuales deban adaptarse a este contexto. Los estudiantes deberían trabajar desde su formación teniendo esta realidad presente en el diseño de sus proyectos profesionales.

Finalmente, hay que señalar que este proyecto se trata de una experiencia docente replicable. Si bien es cierto que no es habitual que los docentes del área de la comunicación (Comunicación Audiovisual, Periodismo y Publicidad y Relaciones Públicas) tengan conocimientos de programación de aplicaciones móviles, creemos que un acuerdo con las facultades de informática podría convertir este proyecto en colaborativo. Un trabajo en equipo entre estudiantes de audiovisuales y estudiantes de ingeniería informática podría permitir que los primeros diseñaran contenidos que serían programados por los segundos. De esta manera, el proyecto de alfabetización transmediática aquí presentado podría ampliarse a la creación de una aplicación móvil por proyecto y en distintos sistemas operativos.

\section{Referencias}

- Andreu-Sánchez, C. \& Martín-Pascual, M.A. (2014a). "La narrativa transmedia audiovisual a través de plataformas móviles” (153-168). En Sierra Sánchez, J. \& García García, F. (coord.). Tecnología y narrativa audiovisual. Madrid: Fragua.

- Andreu-Sánchez, C. \& Martín-Pascual, M.A. (2014b). "La educación audiovisual y la creación de prosumidores mediáticos. Estudio de caso”. adComunica. Revista de Estrategias, Tendencias e Innovación en Educación, 7, 131147.

- Aparici, R. (2010). Introducción: la educomunicación más allá del 2.0. En R. Aparici (Ed.) Educomunicación: más allá del 2.0 (9-23). Barcelona: Gedisa.

- Apple (2014). iOS Human Interface Guidelines. Edición del 10 de marzo de 2014. Recuperado el 14/03/14 https: / / developer.apple.com/library/ios/documenta tion/userexperience/conceptual/MobileHIG/index.h tml.
- Azevedo, H. \& Sánchez, P. (2013). Spain Digital Future in Focus. El Mercado Digital Español. Comscore, abril 2013. Recuperado el 6/03/14

http://www.comscore.com/esl/Insights/Presentation $\underline{s}$ and Whitepapers/2013/2013 Spain Digital Futur e in Focus

- Bisquerra, R. (1989). Métodos de investigación educativa. Guía práctica. Barcelona: CEAC.

- Branda, L.A. (2009). "El aprendizaje basado en los problemas. ¿Un inicio del aprendizaje a lo largo de la vida?" DPM. Avances en Desarrollo Profesional Continuo en Medicina, 2 (4), 15-21.

- Freitas, C. \& Castro, C. (2010). "Narrativas audiovisuales y tecnologías interactivas". Revista Estudios Culturales, (5), 19-42. 
- Giurgiu, L. \& Bârsan, G. (2008). "The prosumer - core and consequence of the Web 2.0 era”. Journal of Social Informatics, año $\mathrm{V}, \mathrm{n}^{\circ}$ 9, 53-59.

- Hernández, A.A. (2014). "Narrativas transmediaticas en entornos digitales: la novela hipermedia Inanimate Alice y sus aplicaciones docentes". CIC. Cuadernos de Información y Comunicación, 19, 287-301.

- Islas, O. (2010). Internet 2.0: El territorio digital de los prosumidores. Revista Estudios Culturales (5), 43-64.

- iTunes (2014). Aplicación Transmedia para iPhone, iPod touch y iPad en el App Store de iTunes. Neurocinematics. Recuperado 14/05/14

https://itunes.apple.com/us/app/transmedia/id7252 $22088 ? \mathrm{l}=\mathrm{es} \& \mathrm{mt}=8$

- Jenkins, H. (2003, Enero 15). Transmedia Storytelling. MIT Technology Review. Recuperado el 28/02/14 http: / / www.technologyreview.com/news/401760/t ransmedia-storytelling/

- Kaplún, M. (2010). Una pedagogía de la comunicación. En R. Aparici (Ed.) Educomunicación: más allá del 2.0 (41-61). Barcelona: Gedisa.

- Klein, N. (1999). No Logo. Canada: Knopf.

- Kotler, Philip (1986). The Prosumer Movement: a New Challenge For Marketers. NA - Advances in Consumer Research Volume 13, eds. Richard J.Lutz, Provo, UT: Association for Consumer Research, 13, 510513.

- Lewin, K. (1946). Action Research and Minority Problems. Journal od Social Issues, 2 (4), 34-46.

- Martos Núñez, E. \& Martos García, A. (2012). "Lecturas fantásticas y Transliteracidad”. Revista Profissão Docente, 12 (26), 71-98.

- Peñalba García, M., Pérez Bowie, J.A. \& Noguerol Jiménez, F. (2013). "Intermedialidad e intertextualidad en la narrativa gráfica”. MID. Memorias de Innovación Docente, 2012-2013. Salamanca: Universidad de Salamanca.
- Proyecto Alien Talkie (2014). Recuperado 25/05/14 http://alientalkie.wordpress.com

- Proyecto Cinetik System (2014). Recuperado 23/05/14 http:// cinetiksystem.tk

- Proyecto ¿Qué vas a comer hoy? (Instagram) (2014). Recuperado el 25/05/14 http: / /instagram.com/quevasacomerhoy

- Quiroz Velasco, M.T. (2010). Educar en otros tiempos. El valor de la comunicación. En R. Aparici (Ed.) Educomunicación: más allá del 2.0 (187-203). Barcelona: Gedisa.

- Scolari, C. (2010). Interfaces para saber, interfaces para hacer. Las simulaciones digitales y las nuevas formas de conocimiento. En R. Aparici (Ed.) Educomunicación: más allá del 2.0 (225-250). Barcelona: Gedisa.

- Scolari, C.A. (2013). Narrativas transmedia. Barcelona: Deusto.

- Suárez Puerta, B.L. (2009). "Reflexiones sobre la enseñanza en situaciones transmediales". Revista Facultad de Ciencias Económicas: Investigación y Reflexión, 17 (2), 183-198.

- The App Date (2013, Septiembre). Informe APPS Septiembre 2013. Madrid The ApP Date. Recuperado 6/03/14 http://madrid.theappdate.com/informeapps-2013/

- Toffler, A. (1980). The Third Wave. Nueva York: William Morrow.

- Toffler, A. \& Toffler, H. (2006). Revolutionary Wealth. USA: Knopf.

- UAB Campus Media (2014). Projecte Transmèdia. Facultat de Comunicació de la Universitat Autònoma de Barcelona. Recuperado el 14/05/14 http: / / www.uab.cat/campusmedia/a-lacarta?serial id $=555$.

\section{Forma de Citación}

ANDREU-SÁNCHEZ, Celia y MARTÍN-PASCUAL, Miguel Ángel: El Transmedia Audiovisual en el aula universitaria. Revista Communication Papers, $\mathrm{N}^{\circ}$ 4, páginas 69 a 76. Departamento de Filología y Comunicación de la Universidad de Girona. Recuperado el __ de de 2 de: http://www.communicationpapers.es 